Georgian Mathematical Journal

Volume 12 (2005), Number 2, 207-216

\title{
GELFAND PAIRS AND GENERALIZED D'ALEMBERT'S AND CAUCHY'S FUNCTIONAL EQUATIONS
}

\author{
BELAID BOUIKHALENE AND SAMIR KABBAJ
}

\begin{abstract}
We show that Cauchy's, d'Alembert's functional equations and their generalizations are the functional equations for bounded spherical functions associated to some Gel'fand pairs. Our results are very close to the ones obtained by Stetkær in [17].
\end{abstract}

2000 Mathematics Subject Classification: 39B32, 39B42, 22D10, 22D12, 22D15.

Key words and phrases: Functional equations, Gel'fand pairs, spherical functions.

\section{INTRODUCTION}

Set-up 1: We adapt here our point of view to the results obtained by Stetkær H. in [17] and [18]. Let $G$ be a locally compact group, and let $K$ be a compact subgroup of $G$ such that $(G, K)$ is a Gel'fand pair ([9] and [11]), the associated $K$-spherical functions are non-zero continuous functions on $G$ such that $\int_{K} f(x k y) d \omega_{K}(k)=f(x) f(y), x, y \in G([9]$ and [11]). Let $K \propto G$ be the semidirect product group, then $(K \propto G, K)$ is a Gel'fand pair (see [5]), and the associated $K$-spherical functions satisfy the functional equation

$$
\int_{K} f(x k \cdot y) d \omega_{K}(k)=f(x) f(y), \quad x, y \in G,
$$

where $\omega_{K}$ is the normalized Haar measure of $K$. If $G$ is an abelian locally compact group and $K$ is a compact subgroup of $\operatorname{Aut}(G)$ consisting of automorphisms and homemorphisms of $G$, then $(K \propto G, K)$ is a Gel'fand pair and the associated bounded $K$-spherical functions satisfy the functional equation

$$
\int_{K} f(x+k \cdot y) d \omega_{K}(k)=f(x) f(y), \quad x, y \in G,
$$

and have the form

$$
f(x)=\int_{K} \chi(k \cdot x) d \omega_{K}(k), \quad x \in G
$$

for some unitary character $\chi$ of $G([3],[8])$. For the Gel'fand pair $(\{I\} \propto G,\{I\})$, the $\{I\}$-spherical functions are solutions of Cauchy's functional equation

$$
f(x+y)=f(x) f(y), \quad x, y \in G .
$$


Another interesting instance is the case of the Gel'fand pair $(\{I,-I\} \propto$ $G,\{I,-I\})$, where the $\{I,-I\}$-spherical functions are solutions of d'Alembert's functional equation

$$
f(x+y)+f(x-y)=2 f(x) f(y), \quad x, y \in G .
$$

In the next set-up, we generalize the results mentioned above.

Set-up 2: Let $G$ be a locally compact group and let $K$ be a compact subgroup of $G$ such that $(G, K)$ is a Gel'fand pair ([5], [8], [10]). Let $\Phi$ be a finite subgroup of $\operatorname{Aut}(G)$ such that $\varphi(K) \subset K, \forall \varphi \in \Phi$. The number of the elements of a finite group $\Phi$ will be denoted by $|\Phi| . C(G)$ (resp. $C_{b}(G)$ ) denotes the space of continuous (resp. continuous and bounded) complex-valued functions. By $L_{1}(G)$ we denote the Banach algebra of all integrable functions on $G$ and by $L_{1}(G \backslash \backslash K)$ the subalgebra of functions in $L_{1}(G)$ that are bi- $K$-invariant (i.e. $f(k x)=f(x k)=f(x), x \in G, k \in K)$. One can form a semi-direct product group $G_{1}=\Phi \propto G$ and a compact subgroup $K_{1}=\Phi \propto K$ using the group law

$$
(\varphi, x)(\psi, y)=(\varphi \psi, x \varphi(y)), \quad \varphi, \psi \in \Phi, \quad x, y \in G .
$$

A function $F: \Phi \propto G \rightarrow \mathbb{C}$, that is bi- $\Phi \propto K$-invariant, can be regarded as a function $f_{\Phi}$ on $G$ such that $f_{\Phi}$ is both bi- $K$-invariant and $\Phi$-invariant (i.e. $f \circ \varphi=\varphi, \forall \varphi \in \Phi)$, therefore $F(\varphi, x)=f_{\Phi}(x), \varphi \in \Phi, x \in G$. Accordingly, we obtain the isomorphism

$$
\begin{aligned}
L_{1}(\Phi \propto G \backslash \backslash \Phi \propto K) & \longrightarrow L_{1}(G \backslash \backslash K) \cap L_{1}^{\Phi}(G), \\
F & \mapsto f_{\Phi},
\end{aligned}
$$

where $L_{1}^{\Phi}(G)=\left\{f \in L_{1}(G): f \circ \varphi=f, \varphi \in \Phi\right\}$. Then $(\Phi \propto G, \Phi \propto K)$ is a Gel'fand pair. Let $x_{1}, y_{1} \in G_{1}=\Phi \propto G$ and let $k_{1} \in K_{1}=\Phi \propto K$, then we have

$$
x_{1} k_{1} y_{1}=(\theta, x)(\varphi, k)(\psi, y)=(\theta \varphi \psi, x \theta(k) \theta(\varphi(y))) .
$$

Let $F \in C_{b}(\Phi \propto G) \backslash\{0\}$, then $F$ is a $\Phi \propto K$-spherical function if and only if $f_{\Phi}$ satisfies the functional equation

$$
\Sigma_{\varphi \in \Phi} \int_{K} f(x k \varphi(y)) d \omega_{K}(k)=|\Phi| f(x) f(y), \quad x, y \in G .
$$

This equation generalizes the following functional equations:

- the $K$-spherical functional equation

$$
\int_{K} f(x k y) d \omega_{K}(k)=f(x) f(y), \quad x, y \in G ;
$$

- the generalized d'Alembert's functional equation

$$
\int_{K} f(x k y) d \omega_{K}(k)+\int_{K} f(x k \sigma(y)) d \omega_{K}(k)=2 f(x) f(y) \quad x, y \in G,
$$

where $\sigma \in \operatorname{Aut}(G)$ is such that $\sigma \circ \sigma=\sigma$ (see [1] and [10]); 
- the Cauchy's functional equation (1.4) and the d'Alembert's functional equations (1.5) and

$$
f(x+y)+f(x+\sigma(y))=2 f(x) f(y) \quad x, y \in G,
$$

where $\sigma \in \operatorname{Aut}(G)$ is such that $\sigma \circ \sigma=\sigma$ (see [19]).

In the previous paper [7], we have dealt with the stability of the functional equation (1.6). Here we study the properties of solutions of the functional equation (1.6). The results obtained generalize the ones obtained in [10], [17], [18] and [19].

In the first section, we give general properties in Proposition 2.3, we prove that if a measurable and essentially bounded function $f$ on $G$ (i.e., $f \in L^{\infty}(G)$ ) satisfies the functional equation (1.6), then $f \in C_{b}(G)$, which explains why we restrict our-selves to solutions $f \in C_{b}(G)$. In the second section, we give a description of the bounded solutions of (1.6). These solutions are expressed by the formula

$$
f(x)=\frac{1}{|\Phi|} \Sigma_{\varphi \in \Phi} l(\varphi(x)), \quad x \in G,
$$

where $l$ is a bounded $K$-spherical function associated to the Gel'fand pair $(G, K)$.

In Section 3, we consider a Riemannian symmetric pair $(G, K)([12])$ and characterize solutions of the functional equation (1.6) as eigenfunctions for some invariant operators on $G$.

In the last section we give some applications of our study.

\section{General Properties}

In what follows, we study general properties of solutions of (1.6). Let $G$ be a locally compact group, $K$ be a compact subgroup of $G$, and let $\Phi$ be a finite group of $K$-invariant automorphisms of $G$.

Proposition 2.1 (7). For an arbitrary fixed $\tau \in \Phi$, and $f \in C(G)$, we have

i) the mapping $\varphi \longrightarrow \varphi \circ \tau$ is an automorphism of $\Phi$;

ii) $\Sigma_{\varphi \in \Phi} \int_{K} f(x k \varphi(\tau(y))) d \omega_{K}(k)=\Sigma_{\varphi \in \Phi} \int_{K} f(x k \varphi(y)) d \omega_{K}(k) ; x, y \in G$.

iii) If $f$ is bi-K-invariant, then for all $z, y, x \in G$, we have

$$
\int_{K} f(y k x) d \omega_{K}(k)=\int_{K} f(x k y) d \omega_{K}(k),
$$

and

$$
\int_{K} \int_{K} f(z h y k x) d \omega_{K}(h) d \omega_{K}(k)=\int_{K} \int_{K} f(z h x k y) d \omega_{K}(h) d \omega_{K}(k) .
$$

Proposition 2.2. Let $f \in C(G) \backslash\{0\}$ be a solution of (1.6), then we have $f(e)=1, f \circ \tau=f$ for all $\tau \in \Phi$ and $f$ is bi-K-invariant.

Proof. By easy computation.

Proposition 2.3. Let $f \in L_{1}^{\infty}(G)$ be a measurable and essentially bounded function on $G$ satisfying the functional equation (1.6), then $f \in C_{b}(G)$. 
Proof. For all $h \in L_{1}(G \backslash \backslash K)$ and for almost all $y \in G$, we have

$$
\begin{aligned}
|\Phi|\langle\check{h}, f\rangle f(y)=|\Phi| \int_{G} f(y) \check{h}(x) f(x) d x \\
=\Sigma_{\varphi \in \Phi} \int_{K} \int_{G} f(x k \varphi(y)) h\left(x^{-1}\right) d \omega_{K}(k) d x \\
=\Sigma_{\varphi \in \Phi} \int_{K} \int_{G} f\left(x^{-1} k \varphi(y)\right) h(x) d \omega_{K}(k) d x \\
=\Sigma_{\varphi \in \Phi} \int_{K}(h * f)(k \varphi(y)) d \omega_{K}(k)=\Sigma_{\varphi \in \Phi}(h * f)(\varphi(y))
\end{aligned}
$$

which finishes the proof of our proposition.

\section{Solutions of Equation (1.6)}

In the following theorem, we determine the bounded solutions of the functional equation (1.6).

Theorem 3.1. Let $G$ be a locally compact group and let $K$ be a compact subgroup such that $(G, K)$ is a Gel'fand pair. Let $\Phi$ be a finite subgroup of the group Aut $(G)$ such that $K$ is $\Phi$-invariant. Let $f \in C_{b}(G) \backslash\{0\}$. Then $f$ is a solution of equation (1.6) if and only if there exists a $K$-spherical function $l$ such that

$$
f(x)=\frac{1}{|\Phi|} \Sigma_{\varphi \in \Phi} l(\varphi(x)), \quad x \in G .
$$

Proof. By using some ideas of [7] and [2], we define for each fixed $y \in G$, the function $\chi_{y}$ on $\Sigma$ (the Gel'fand spectrum of the commutative Banach algebra $L_{1}(G \backslash \backslash K)$ (see [11]) to be

$$
\chi_{y}(l)=\frac{1}{|\Phi|} \Sigma_{\varphi \in \Phi} l(\varphi(y)), \quad l \in \Sigma .
$$

Let $F\left(L_{1}(G \backslash \backslash K)\right)$ be the set of Fourier transforms of functions in $L_{1}(G \backslash \backslash K)$. For all $g \in F\left(L_{1}(G \backslash \backslash K)\right)$, we have $\chi_{y} g \in F\left(L_{1}(G \backslash \backslash K)\right)$. Indeed, if $g=F h$, $h \in L_{1}(G \backslash \backslash K)$, then $\chi_{y} g=F z$, where $z \in L_{1}(G \backslash \backslash K)$ has the form

$$
z(x)=\frac{1}{|\Phi|} \Sigma_{\varphi \in \Phi} \int_{K} h\left(x k \varphi\left(y^{-1}\right)\right) d \omega_{K}(k) .
$$

Now let $F f$ (the Fourier transform of $f$ ) be regarded as a pseudomeasure on $L_{1}(G \backslash \backslash K)$ (see [5]) defined by

$$
\langle F f, g\rangle=\int_{G} f(x) h(x) d x,
$$

where $F h=g$. We show that for each $y \in G$ and $g \in F\left(L_{1}(G \backslash \backslash K)\right)$,

$$
\left[\left(\chi_{y}-f(y)\right) g\right] F f=0 .
$$


Indeed, for each $g \in F\left(L_{1}(G \backslash \backslash K)\right)$, we have

$$
\left\langle\left[\left(\chi_{y}-f(y)\right) g\right] F f, A\right\rangle=\int_{G} f(x)(z * q)(x) d x-f(y) \int_{G} f(x)(h * q)(x) d x,
$$

where $F h=g, F q=A$ and $F z=\chi_{y}(g)$. Then we obtain

$$
\begin{aligned}
\left\langle\left[\left(\chi_{y}-\right.\right.\right. & f(y)) g] F f, A\rangle=\frac{1}{|\Phi|} \int_{G} \int_{K} f(x) \Sigma_{\varphi \in \Phi}(h * q)\left(x \varphi\left(y^{-1}\right) k\right) d \omega_{K}(k) d x \\
& -f(y) \int_{G} f(x)(h * q)(x) d x \\
= & \frac{1}{|\Phi|} \int_{G} \int_{K} f(x) \Sigma_{\varphi \in \Phi}(h * q)\left(x k \varphi\left(y^{-1}\right)\right) d \omega_{K}(k) d x \\
& -f(y) \int_{G} f(x)(h * q)(x) d x \\
= & \frac{1}{|\Phi|} \int_{G} \int_{K} f(x) \Sigma_{\varphi \in \Phi}(h * q)(x k \varphi(y)) d \omega_{K}(k) d x \\
& -f(y) \int_{G} f(x)(h * q)(x) d x \\
= & f(y) \int_{G} f(x)(h * q)(x) d x-f(y) \int_{G} f(x)(h * q)(x) d x=0 .
\end{aligned}
$$

Since $f$ is non-zero, the support of the pseudomeasure $F f$ is nonempty. Let $l$ be in the support of $F f$. Then we obtain that, for all $y \in G$ and for all $g \in F\left(\left(L_{1}(G \backslash \backslash K)\right)\right.$, the function $\left(\chi_{y}-f(y)\right) g$ vanishes at $l$ (see [5] Theorem 1.3.1). Consequently, we have $\chi_{y}(l)=f(y)$, which ends the proof.

\section{On Riemannian Symmetric Pairs}

In this section, we suppose that $G$ is a connected compact Lie group and $K$ is a compact subgroup of $G$ such that $(G, K)$ is a Riemannian symmetric pair, then $(G, K)$ is a Gel'fand pair ([13]). Let $\Phi$ be a finite subgroup of Aut $(G)$ such that $\varphi(K) \subset K$ for all $\varphi \in \Phi$. We shall characterize solutions of (1.6) in terms of eigenfunctions of some left invariant differential operators.

For each fixed $a \in G$, we define the left (resp. the right) translation operators as $\left(L_{a} f\right)(x)=f\left(a^{-1} x\right)$ (resp. $\left.\left(R_{a} f\right)(x)=f(x a)\right)$ and say that an operator $T$ is left (resp. right) invariant if $\left(L_{a} T\right) f=T\left(L_{a} f\right)$ (resp. $\left.\left(R_{a} T\right) f=T\left(R_{a} f\right)\right)$. Let $\mathbb{D}(G)$ denote the algebra of left invariant differential operators on $G$. For all $f \in C(G)$, one puts $f^{\natural}(x)=\int_{K} \int_{K} f(k x h) d \omega_{K}(k) d \omega_{K}(h)$, for all $x \in G$. 

by

Proposition 4.1. For any differential operator $D$, the operator $D_{K}$ defined

$$
\left(D_{K} f\right)(x)=\frac{1}{|\Phi|} \Sigma_{\varphi \in \Phi} D\left\{\left(L_{x^{-1}} f\right)^{\natural} \circ \varphi\right\}(e),
$$

for all $f \in C^{\infty}(G)$ and $x \in G$, has the following properties:

i) $D_{K}$ is left invariant;

ii) $D_{K}$ is right invariant under $K$. Furthermore one has $R_{h} D_{K}=D_{K} R_{h}=$ $D_{K}$

iii) $\left(D_{K} f\right)(e)=\frac{1}{|\Phi|} \Sigma_{\varphi \in \Phi} D\left\{f^{\natural} \circ \varphi\right\}(e)$. In particular if $f$ is bi-K-invariant and $f \circ \tau=f$ for all $\tau \in \Phi$ then $\left(D_{K} f\right)(e)=(D f)(e)$;

vi) If $f$ is a solution of $(1.6)$, then $D_{K} f=(D f)(e) f$.

Proof. By easy computations, we have iii) and vi).

For i) let $f \in C^{\infty}(G)$ and let $a \in G$, for all $x \in G$, we have

$$
\begin{aligned}
L_{a}\left(D_{K} f\right)(x) & \left.=\left(D_{K} f\right)\left(a^{-1} x\right)=\frac{1}{|\Phi|} \Sigma_{\varphi \in \Phi} D\left\{\left(L_{\left(x^{-1} a\right.}\right) f\right)^{\natural} \circ \varphi\right\}(e) \\
& =\frac{1}{|\Phi|} \Sigma_{\varphi \in \Phi} D\left\{\left(L_{x^{-1}}\left(L_{a} f\right)\right)^{\natural} \circ \varphi\right\}(e)=D_{K}\left(L_{a} f\right)(x) .
\end{aligned}
$$

For ii) let $f \in C^{\infty}(G)$ and let $h \in K$, for all $x \in G$, we have

$$
\begin{aligned}
R_{h}\left(D_{K} f\right)(x) & =\left(D_{K} f\right)(x h)=\frac{1}{|\Phi|} \Sigma_{\varphi \in \Phi} D\left\{\left(L_{(x h)^{-1}} f\right)^{\natural} \circ \varphi\right\}(e) \\
& =\frac{1}{|\Phi|} \Sigma_{\varphi \in \Phi} D\left\{\left(L_{x^{-1}}\left(R_{h} f\right)\right)^{\natural} \circ \varphi\right\}(e)=D_{K}\left(R_{h} f\right)(x) .
\end{aligned}
$$

For vi)

$$
\begin{aligned}
\Sigma_{\varphi \in \Phi}\left(L_{x^{-1}} f\right)^{\natural}(\varphi(y)) & =\Sigma_{\varphi \in \Phi} \int_{K}\left(L_{x^{-1}} f\right)(k \varphi(y)) d \omega_{K}(k) \\
& =\Sigma_{\varphi \in \Phi} \int_{K} f(x k \varphi(y)) d \omega_{K}(k)=|\Phi| f(x) f(y) .
\end{aligned}
$$

For $y=e$ we get

$$
\left(D_{K} f\right)(x)=\frac{1}{|\Phi|} \Sigma_{\varphi \in \Phi} D\left\{\left(L_{x^{-1}} f\right)^{\natural} \circ \varphi\right\}(e)=(D f)(e) f(x) .
$$

Lemma 4.1. If $f$ is a solution of equation (1.6), then $f$ is analytic.

Proof. Since $F$ is a $\Phi \propto K$-spherical function and the space $\Phi \propto G \backslash \Phi \propto G$ is connected, using the proof in [13] we derive the rest.

Theorem 4.1. Let $G$ be a connected Lie group and let $K$ be a compact subgroup of $G$ such that $(G, K)$ is a Rimannian symmetric pair. Let $\Phi$ be a finite subgroup of $\operatorname{Aut}(G)$ such that $K$ is $\Phi$-invariant. Let $f \in C(G)$. Then the following statements are equivalent:

(1) $f$ is a solution of (1.6).

$(2) \quad$ i) $f(e)=1, f \circ \tau=f$ for all $\tau \in \Phi$ and $f$ is bi-K-invariant; 
ii) $f \in C^{\infty}(G)$;

iii) $f$ is analytic;

vi) $f$ is a joint eigenfunction of the operators $D_{K}$ for all $D \in \mathbb{D}(G)$.

Proof. (1) $\Longrightarrow$ (2) follows directly from Propositions 4.1 and 2.2 and Lemma 4.1. Conversely, suppose that $D_{K} f=(D f)(e) f$ for all $D \in \mathbb{D}(G)$. For a fixed $x \in G$ we define the function

$$
F(y)=\frac{1}{|\Phi|} \Sigma_{\varphi \in \Phi} \int_{K} f(x k \varphi(y)) d \omega_{K}(k), \quad y \in G .
$$

Since $f \circ \tau=f$, for all $\tau \in \Phi$ we get

$$
F(y)=\frac{1}{|\Phi|} \Sigma_{\varphi \in \Phi} \int_{K}\left(L_{(\varphi(x) k)^{-1}} f\right)(y) d \omega_{K}(k) .
$$

Consequently, for all $D \in \mathbb{D}(G)$ we have

$$
\left(D_{K} F\right)(y)=\frac{1}{|\Phi|} \Sigma_{\varphi \in \Phi} \int_{K} D_{K}\left(L_{(\varphi(x) k)^{-1}} f\right)(y) d \omega_{K} .
$$

Since $D_{K}$ is left invariant, we obtain

$$
\left(D_{K} F\right)(y)=D f(e) F(y) .
$$

In particular for $y=e$ we have

$$
\left(D_{K} F\right)(e)=D f(e) F(e)
$$

Hence, by Proposition 4.1 iii), it follows that

$$
(D F)(e)=D(f)(e) F(e)
$$

i.e.,

$$
D(F-F(e) f)(e)=0
$$

for all $D \in \mathbb{D}(G)$.

Since $F-F(e) f$ is an analytic function on the connected Lie group $G$, by [13] we obtain

$$
F-F(e) f \equiv 0
$$

on $G$. We conclude that

$$
\frac{1}{|\Phi|} \Sigma_{\varphi \in \Phi} \int_{K} f(x k \varphi(y)) d \omega_{K}(k)=f(x) f(y), \quad x, y \in G .
$$

\section{Applications}

5.1. Let $G$ be an abelian, locally compact group and let $\Phi$ be a finite subgroup of $\operatorname{Aut}(G)$, then $(\Phi \propto G, \Phi)$ is a Gel'fand pair and the associated bounded $\Phi$ spherical functions are solutions of the functional equation

$$
\Sigma_{\varphi \in \Phi} f(x+\varphi(y))=|\Phi| f(x) f(y), \quad x, y \in G,
$$


and have the form

$$
f(x)=\frac{1}{|\Phi|} \Sigma_{\varphi \in \Phi} \chi(\varphi(x)), \quad x \in G
$$

for some unitary character $\chi$ of $G$.

If $\sigma: G \longrightarrow G$ is a continuous automorphism for $G$ such that $\sigma \circ \sigma=I$, then the bounded $\{I, \sigma\}$-spherical functions associated to the Gel'fand pair $(\{I, \sigma\} \propto G,\{I, \sigma\})$ satisfy d'Alembert's functional equation

$$
\frac{f(x+y)+f(x+\sigma(y))}{2}=f(x) f(y), \quad x, y \in G,
$$

and have the form

$$
f=\frac{\chi+\chi \circ \sigma}{2}
$$

for some character $\chi$ of $G$ (see [4], [8], [14], [15] and [18]).

5.2. Let $(G, K)$ be a compact Gel'fand pair and let $\Phi$ be a finite subgroup of $\operatorname{Aut}(G)$ such that $\varphi(K) \subset K$ for all $\varphi \in \Phi$. Then the $\Phi \propto K$-spherical functions associated to the compact Gel'fand pair $(\Phi \propto G, \Phi \propto K)$ are solutions of the functional equation (1.6) and each bounded one has the form

$$
f(x)=\frac{1}{|\Phi|} \Sigma_{\varphi \in \Phi}\langle\pi(x) \xi, \xi\rangle, \quad x \in G,
$$

where $\left(\pi, H_{\pi}\right)$ is an irreducible continuous and unitary representation on a Hilbert space $H_{\pi},\langle$,$\rangle is the inner product of the space H_{\pi}$ and $\xi$ is a unit vector satisfying $\pi(k) \xi=\xi$ for all $k \in K$ (see [1]).

5.3. Let $G$ be a noncompact connected semi-simple Lie group with finite center and $K$ be a maximal compact subgroup of $G$. Then $(G, K)$ is a Gel'fand pair [9]. By Iwasawa decomposition, we may write $G=K S$, where $S$ is a closed solvable subgroup. Let $\Phi$ be a finite subgroup of $\operatorname{Aut}(G)$. Then the bounded $\Phi \propto K$-spherical functions associated to the Gel'fand pair $(\Phi \propto G, \Phi \propto K)$ are solutions of the functional equation (1.6) and have the form

$$
f(x)=\frac{1}{|\Phi|} \Sigma_{\varphi \in \Phi} \int_{K} \chi(k \varphi(x)) d \omega_{K}(k), \quad x \in G,
$$

for some unitary character $\chi$ of $S$.

5.4. Let $G$ be a unimodular group containing a normal commutative group $A$ with no elements of order 2 and let $K$ be a nonnormal compact subgroup of $G$. Then $(G, K)$ is a Gel'fand pair [9]. Let $\Phi$ be a finite subgroup of $\operatorname{Aut}(G)$. Then the bounded $\Phi \propto K$-spherical functions associated to the Gel'fand pair $(\Phi \propto G, \Phi \propto K)$ are solutions of the functional equation (1.6) and have the form

$$
f(x)=\frac{1}{|\Phi|} \Sigma_{\varphi \in \Phi} \int_{K} \chi\left(k \varphi(s) k^{-1}\right) d \omega_{K}(k), \quad x \in G,
$$

where $x=t s, t \in K, s \in A$ and $\chi$ is a unitary character of $A$.

5.5. Let $G$ be a locally compact group group and let $K$ be a compact subgroup of $G$ such that $(G, K)$ is a Gel'fand pair. Let $\sigma: G \longrightarrow G$ be a continuous 
automorphism of order 2 such that $\sigma(K) \subset K$. Then $(\{I, \sigma\} \propto G,\{I, \sigma\} \propto K)$ is a Gel'fand pair and the associated bounded $\{I, \sigma\} \propto K$-spherical functions are solutions of the functional equation

$$
\int_{K} f(x k y) d \omega_{K}(k)+\int_{K} f(x k \sigma(y)) d \omega_{K}(k)=2 f(x) f(y), \quad x, y \in G,
$$

and have the form

$$
f=\frac{\omega+\omega \circ \sigma}{2}
$$

for some bounded $K$-spherical function $\omega$ on $G$ (see [1] and [10]).

\section{ACKNOWLEDGEMENT}

We are very grateful to Professor H. Stetkær for discussions and his many useful comments and remarks.

\section{REFERENCES}

1. M. Akkouchi, A. Bakali, and E. Elqorachi D'Alembert's functional equation on compact Gel'fand pairs. Maghreb Math. Rev. 8(1999), No. 1-2, 1-9.

2. M. Akkouchi, A. Bakali, B. Bouikhalene, and E. Elqorachi, On a generalized of a class of functional equations on a locally compact group. Submitted

3. R. BADORA, On a joint generalization of Cauchy's and d'Alembert's functional equations. Aequationes Math. 43(1992), No. 1, 72-89.

4. J. A. Baker, The stability of the cosine equation. Proc. Amer. Math. Soc. 80(1980), No. 3, 411-416.

5. J. J. Benedetto, Spectral synthesis. Mathematische Leitfäden. B. G. Teubner, Stuttgart, 1975.

6. C. Benson, J. Jenkins, and G. Ratcliff, On Gel'fand pairs associated with solvable Lie groups. Trans. Amer. Math. Soc. 321(1990), No. 1, 85-116.

7. B. Bouikhalene, On the stability of a class of functional equations. JIPAM. J. Inequal. Pure Appl. Math. 4(2003), No. 5, Article 104, 8 pp. (electronic).

8. W. ChOJNACKI, On some functional equation generalizing Cauchy's and d'Alembert's functional equations. Colloq. Math. 55(1988), No. 1, 169-178.

9. J. Dieudonné, Éléments d'analyse. Tome VI. Chapitre XXII. Cahiers Scientifiques, Fasc. XXXIX. Gauthier-Villars Éditeur, Paris, 1975.

10. H. Elqorachi and M. Akkouchi, On generalized d'Alembert and Wilson functional equations. Aequationes Math. 66(2003), No. 3, 241-256.

11. J. FAraut, Analyse harmonique sur les paires de Guelfand et les espaces hyperboliques. les Cours du CIMPA, université de Nancy I, 1980.

12. S. Helgason, Differential geometry and symmetric spaces. Pure and Applied Mathematics, Vol. XII. Academic Press, New York-London, 1962.

13. S. Helgason, Groups and geometric analysis. Integral geometry, invariant differential operators, and spherical functions. Pure and Applied Mathematics, 113. Academic Press, Inc., Orlando, FL, 1984.

14. Pl. Kannappan, The functional equation $f(x y)+f\left(x y^{-1}\right)=2 f(x) f(y)$ for groups. Proc. Amer. Math. Soc. 19(1968), 69-74.

15. H. SHIN'YA, Spherical matrix functions and Banach representability for locally compact motion groups. Japan. J. Math. (N.S.) 28(2002), No. 2, 163-201. 
16. R. C. Penney and A. L. Rukhin, d'Alembert's functional equation on groups. Proc. Amer. Math. Soc. 77(1979), No. 1, 73-80.

17. H. StetkæR, d'Alembert's equation and spherical functions. Aequationes Math. 48 (1994), No. 2-3, 220-227.

18. H. StetkæR, Harmonic analysis and functional equations. Harmonic analysis and hypergroups (Delhi, 1995), 131-143, Trends Math., Birkhäuser Boston, Boston, MA, 1998.

19. H. StetkæR, Functional equations on abelian groups with involution. Aequationes Math. 54(1997), No. 1-2, 144-172.

(Received 24.04.2004; revised 1.10.2004)

Authors' addresses:

B. Bouikhalene

Department of Mathematics, University of Ibn Tofail

Faculty of Sciences BP 133, Kenitra 14000

Morocco

E-mail: bbouikhalene@yahoo.fr

S. Kabbaj

Department of Mathematics, University of Ibn Tofail

Faculty of Sciences BP 133, Kenitra 14000

Morocco

E-mail: samkabbaj@yahoo.fr 